\title{
SERUM CALCITONIN, THYROTROPIN, AND GOITER
}

\section{GATEN7 Giorgio Grani, Mimma D'Alessandri, Marianna Del Sordo, Giovanni Carbotta, Martina Vitale, Angela Fumarola \\ Dept. of Experimental Medicine - Endocrinology Unit - Sapienza University of Rome}

\section{BACKGROUND}

Recent papers reported that basal calcitonin (CT) level may be related to thyroid volume. This study aims to evaluate if this finding is confirmed in patients undergoing ultrasonography-guided fine-needle aspiration cytology (FNAC) for thyroid nodules.

\section{METHODS}

From February 2010 to September 2012, 561 patients underwent ultrasonography-guided FNAC and a complete evaluation including basal serum FT4, FT3, TSH, CT and estimation of the thyroid volume.

Design: Retrospective university-center study.

\section{RESULTS}

The mean thyroid volume was $21.10 \pm 9.58 \mathrm{~mL}$ in males and $13.42 \pm 6.48 \mathrm{~mL}$ in females $(p<0.001)$. Thyroid was found to be atrophic in 18 cases and goiter was diagnosed in 128 patients. A linear regression analysis was performed between serum CT levels and thyroid volume, showing a weak direct relationship $(\mathrm{R} 2=0.023, \mathbf{p}<\mathbf{0 . 0 0 1})$. There is no correlation between serum TSH and CT levels. In patients grouped according to morphologic diagnosis (atrophy, normal volume and goiter), CT levels are slightly higher in the high-volume groups: the mean value was $2.02 \pm 0.09$ in the atrophy group, $2.86 \pm 1.73$ in the normal volume group, and $3.00 \pm 1.66$ in the goiter group $(p=0.02)$. When males and females are computed separately the statistical significance is lost.

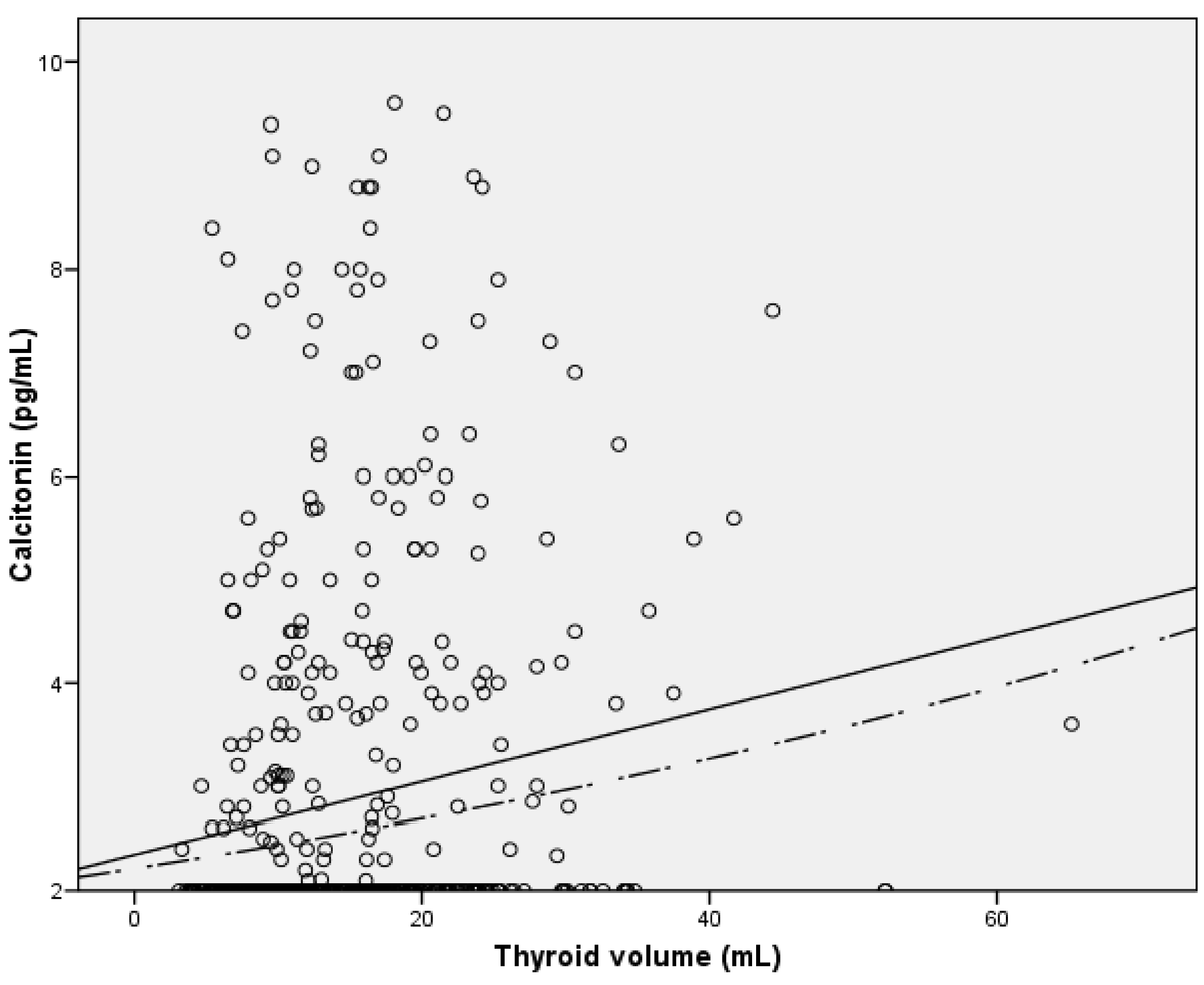

Figure 1: Relationship between CT values and thyroid volume; linear (solid line) and exponential (dashed line) regressions $\left(R^{2}=0.025\right.$ and 0.03 respectively, $\left.p<0.001\right)$

\section{CONCLUSIONS}

The small difference in basal CT levels is probably due to a genetically determined higher thyroid volume and increased number of $\mathrm{C}$-cells rather than to an acquired goiter. Gender may act as a "surrogate marker" of thyroid volume and the application of a gender-specific cut-off can probably overcome this issue.
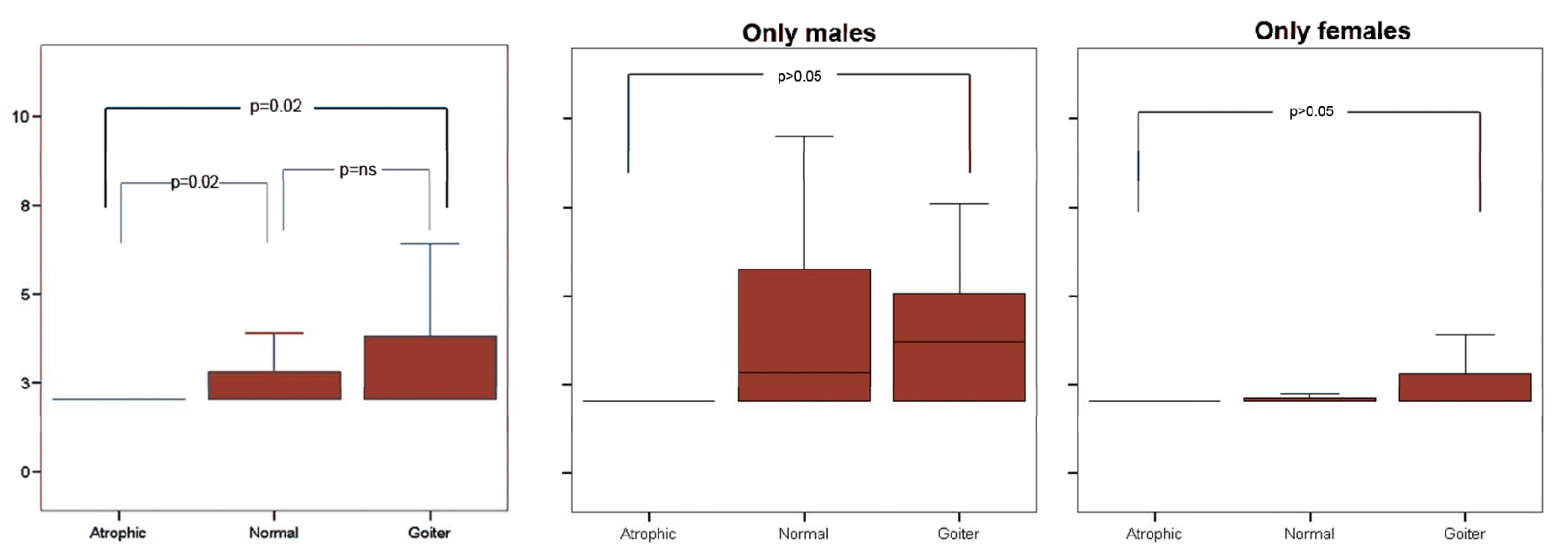

\section{REFERENCES}

Figure 2: Median serum CT value in patients grouped by thyroid volume. The box represents the interquartile range. The line across the box marks the median.

Glands with an estimated volume $<4.5 \mathrm{~mL}$ (females) and $<5.5 \mathrm{~mL}$ (males) were considered atrophic; meanwhile thyroid volume $>18 \mathrm{~mL}$ in women and $>25 \mathrm{~mL}$ in men, which corresponds to the mean + 3SD in iodine-sufficient population, was diagnosed as goiter

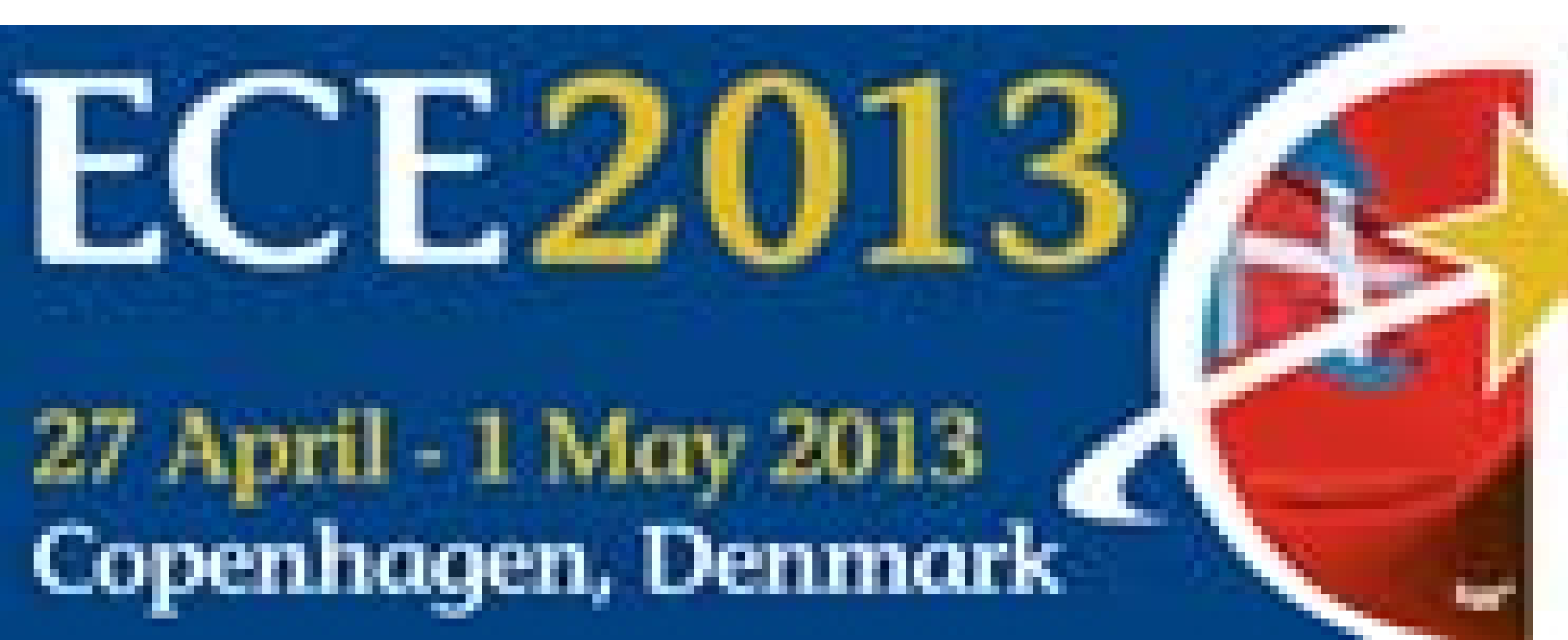

